\title{
Antidepressant-like Effects of Buprenorphine are Mediated by Kappa Opioid Receptors
}

\author{
Edgardo Falcon ',4, Caroline A Browne ${ }^{1,4}$, Rosa M Leon', Vanessa C Fleites', Rachel Sweeney', Lynn G Kirby ${ }^{2}$ \\ and Irwin Lucki*,1,3
}

'Department of Psychiatry, University of Pennsylvania, Philadelphia, PA, USA; ${ }^{2}$ Department of Pharmacology, Temple University School of Medicine, Philadelphia, PA, USA; ${ }^{3}$ Department of Pharmacology, University of Pennsylvania, Philadelphia, PA, USA

\begin{abstract}
Previous studies have identified potential antidepressant effects of buprenorphine (BPN), a drug with high affinity for mu opioid receptor (MORs) and kappa opioid receptors (KORs) and some affinity at delta opioid receptor (DOR) and opioid receptor-like I (ORL-I) receptors. Therefore, these studies examined which opioid receptors were involved in BPN's effects on animal behavior tests sensitive to antidepressant drugs. The acute effects of BPN were tested in the forced swim test (FST) using mice with genetic deletion of individual opioid receptors or after pharmacological blockade of receptors. For evaluating the effects of BPN on chronic stress, separate groups of mice were exposed to unpredictable chronic mild stress (UCMS) for 3 weeks and treated with BPN for at least 7 days before behavioral assessment and subsequent measurement of Oprkl, Oprm I, and Pdyn mRNA expression in multiple brain regions. BPN did not reduce immobility in mice with KOR deletion or after pretreatment with norbinaltorphimine, even though desipramine remained effective. In contrast, BPN reduced immobility in MOR and DOR knockout mice and in mice pretreated with the ORL-I antagonist JTC-80 I. UCMS reduced sucrose preference, decreased time in the light side of the light/dark box, increased immobility in the FST and induced regionspecific alterations in Oprk I, Oprm I, and PDYN mRNA expression in the frontal cortex and striatum. All of these changes were normalized following BPN treatment. The KOR was identified as a key player mediating the effects of BPN in tests sensitive to antidepressant drugs in mice. These studies support further development of BPN as a novel antidepressant.

Neuropsychopharmacology (2016) 4I, 2344-235I; doi:I0.1038/npp.2016.38; published online 20 April 2016
\end{abstract}

\section{INTRODUCTION}

A growing body of evidence points to the kappa opioid receptor (KOR), and its endogenous ligand dynorphin, as key players in the regulation of mood and responses to stress (Lutz and Kieffer, 2013). Acute administration of KOR agonists and antagonists has been shown to produce effects characterized as prodepressive or antidepressant, respectively, in rodent behavioral tests sensitive to antidepressant drugs, (Carlezon et al, 2006; Carr et al, 2010; Mague et al, 2003). Furthermore, exposure to stress is known to sensitize the KOR system, and pretreatment with the selective KOR antagonist norbinaltorphimine (nor-BNI) blocks stressdependent responses (Land et al, 2008; McLaughlin et al, 2003). McLaughlin et al (2003) found that antagonizing KORs can block prodepressive effects produced by exposure to repeated swim stress. These preclinical findings have encouraged further investigation of KOR antagonists as potential antidepressant treatments in humans, particularly

* Correspondence: Dr I Lucki, Department of Psychiatry, University of Pennsylvania, 125 South 31 st Street, Room 2204, Philadelphia, PA 19104-3404, USA, Tel: +I 215573 3305, Fax: + I 215573 2I49, E-mail: lucki@mail.med.upenn.edu

${ }^{4}$ These authors contributed equally to this work.

Received 5 November 2015; revised 2 March 2016; accepted 5 March 2016; accepted article preview online 16 March 2016 in patients diagnosed with major depressive disorder (MDD) that do not respond to conventional antidepressant medications.

Buprenorphine (BPN) is an opioid drug with mixed pharmacology, approved by the US Food and Drug Administration for treatment of heroin dependence and chronic pain. Its most potent pharmacological actions are as a high-affinity partial agonist at the mu opioid receptor (MOR) and antagonist at the KOR. In addition, at higher concentrations, BPN is an antagonist at the delta opioid receptor (DOR) and a partial agonist at the opioid receptorlike 1 (ORL-1) receptor (Cowan, 2007; Lutfy and Cowan, 2004). In a handful of clinical studies, low doses of BPN produced impressive alleviation of depressive symptoms in patients that were considered treatment-resistant within 1 week of initiating administration (Bodkin et al, 1995; Nyhuis et al, 2008). Subsequently, a study with geriatric treatment-resistant MDD patients showed a significant mood-elevating effect of BPN after 1 week of treatment (Karp et al, 2014). Moreover, a recent study demonstrated significant antidepressant effects of ALKS5461, a combination of BPN and samidorphan, a MOR antagonist, following just 1 week of treatment in subjects with MDD (Ehrich et al, 2015). In addition, studies in healthy controls have shown that low doses of BPN can reduce the sensitivity to fearful facial expressions (Ipser et al, 2013) and enhanced the 
attention for more positive emotional cues (Syal et al, 2015), suggesting that BPN can modulate emotional valence in the absence of hedonic experiences.

Our laboratory has shown previously that low doses of BPN produced antidepressant and anxiolytic responses in mice and rats when tested $24 \mathrm{~h}$ post administration (Browne et al, 2015; Falcon et al, 2015). The present study further characterizes antidepressant responses to BPN in C57BL/6J mice, a strain that demonstrates a refractory response to SSRIs in the forced swim test (FST) (Balu et al, 2009; Lucki et al, 2001). First, the acute effect of BPN in the FST was evaluated in C57BL/6J mice after pharmacological challenge and in opioid receptor knockout mice to determine which opioid receptor has a primary role in mediating the observed antidepressant response. Second, BPN was evaluated in C57BL/6J mice after exposure to unpredictable chronic mild stress (UCMS), a validated and widely used rodent model of depression that produces behavioral deficits that are reversed only by chronic antidepressant treatment (Willner, 1997).

\section{MATERIALS AND METHODS}

\section{Animals}

Male C57BL/6J mice, 8 weeks old when purchased from Jackson Laboratories (Bar Harbor, ME) were used for the majority of the studies. Male Oprm1 $1^{--}$mice and littermate wild-type (WT) controls were generated using heterozygous breeding. Mating pairs of Oprk1 ${ }^{-/-}$mice were purchased from Jackson Laboratories and the mice used in this study were generated from initial pairings via homozygous breeding. The $O \mathrm{prd1}^{-/-}$mice were from a colony maintained by homozygous breeding and tested at Temple University. Male C57BL/6J mice, originally from Jackson Laboratories but generated within the colony, were used as their WT controls because they are the background strain used in all of the genetic lines. Mice were housed up to five per cage and maintained under a $12 \mathrm{~h}$ light-dark cycle (lights on at 0730 hours) in temperature and humidity-controlled rooms at the University of Pennsylvania. Food and water were freely available. All studies were approved by the Institutional Animal Care and Use Committee for the University of Pennsylvania and conducted according to accepted guidelines such as 'Guiding principles in the care and use of animals' (DHEW Publications, NIH, 80-23).

\section{Drugs}

BPN hydrochloride (Sigma, St Louis, MO and NIDA), norBNI (Tocris Bioscience, Ellisville, MO) and desipramine hydrochloride (DMI; Sigma) were administered at doses previously found to have antidepressant activity in the FST (Falcon et al, 2015; Lucki et al, 2001; McLaughlin et al, 2003). JTC-801 (Tocris) was administered at $1 \mathrm{mg} / \mathrm{kg}$ based on pilot data (unpublished). All compounds were dissolved in distilled water, sonicated for $15 \mathrm{~min}$, and injected intraperitoneally (i.p.). Mice in the control groups were injected with $0.9 \%$ saline. All doses were calculated according to the base weight of the drug and administered in a volume of $10 \mathrm{ml} / \mathrm{kg}$.

\section{FST}

Swim sessions were conducted either $30 \mathrm{~min}$ or $24 \mathrm{~h}$ after treatment and were performed as described previously (Falcon et al, 2015). In brief, mice were placed in individual polycarbonate cylinders $(25.3 \mathrm{~cm}$ tall $\times 22.2 \mathrm{~cm}$ in diameter $)$ filled with water $\left(25 \pm 1^{\circ} \mathrm{C}\right)$ to a depth of $15 \mathrm{~cm}$. Each session lasted $6 \mathrm{~min}$ and water was changed between each animal. Sessions were recorded with a digital camera from an overhead view to allow for manual scoring of immobile behavior. Immobility was defined as the absence of movement, except that necessary to maintain the head above the water. Mice typically adopted this passive immobile posture after actively swimming for $\sim 2 \mathrm{~min}$ in the swim tank. The standard protocol recommends scoring only the last $4 \mathrm{~min}$ of the 6-min test (Porsolt et al, 1977). However, because stress and genetic modifications can change performance during the first 2 min of the FST, immobility during the entire 6min test was scored in all of these studies.

\section{UCMS Procedure}

Mice were exposed to a variable sequence of mild, unpredictable stressors for 3 weeks as previously described with minor modifications (Schmidt and Duman, 2010). These stressors were physical or psychosocial and applied three times per day, in the morning (beginning at 0930 hours), afternoon (beginning at 1430 hours), and overnight in a dedicated procedure room. Mice were returned to the animal colony after exposure to the stressors. The exact stressors and sequence is presented in Supplementary Table 1. During the last week of stress, mice were injected with either saline or BPN $(0.25 \mathrm{mg} / \mathrm{kg}$, i.p.) for 7 days prior to behavioral testing. Mice were subjected to a series of behavioral tests during the next 7 days. After each behavioral test was completed, mice were injected with BPN and tested the following day in order to maintain treatment and avoid any motor effects influencing the behavioral tests.

\section{Sucrose Preference Test}

The sucrose preference test was performed as previously described (Strekalova and Steinbusch, 2010). To reduce variability and neophobia upon first exposure to the sucrose test, mice were permitted to consume $2.5 \%$ sucrose from a single bottle for $2 \mathrm{~h}$ during the dark phase the night prior to testing. During an $8 \mathrm{~h}$ session the next day, mice were given a free choice between two bottles, one with water and one with a $1 \%$ sucrose solution. To avoid side preference, bottles were switched halfway through the test. Fluid intake was determined by the difference in weight of the bottles from before to after the test. Preference was calculated as the percentage of sucrose consumed from the total amount of fluid ingested (sucrose and water). Animals were randomized across groups after establishment of baseline sucrose preference. Sucrose preference of $60 \%$ or higher was used as the cut off criteria for inclusion.

\section{Dark/Light Emergence Test}

The dark/light emergence (DL) test was adapted from previously described procedures (Mozhui et al, 2010). The 
testing apparatus consisted of a two-chambered box $(17 \times 20 \mathrm{~cm}$ for each side) made of Plexiglas with an opening $(5 \times 5 \mathrm{~cm})$ connecting both chambers. One side was colored black (room light entry limited) and the other side was white, illumination was 800 lux. Mice were placed into the dark side and then allowed to freely explore either the light or dark side for $5 \mathrm{~min}$. Anxiety-like behavior was measured as the time spent exploring the 'light' side during the 5 min session. Two animals failed to emerge and were excluded from analysis.

\section{Quantitative RT-PCR}

Brain tissue from the frontal cortex (FC), hippocampus (Hp), striatum (Str), and amygdala (Amy) was collected by gross dissection from two separate cohorts of male C57BL/6J mice exposed to the UCMS procedure. In brief, a Trizol (Life Sciences cat. No 15596-026)/chloroform-based extraction method was used to isolate total RNA. Samples were homogenized using a Kontes Pellet Pestle. Quantification of the isolated RNA was performed using the Nanodrop spectrophotometer and ND-1000 software at the optical densities of 260 and $280 \mathrm{~nm}$. Samples with poor RNA quality/degradation were excluded at this stage. Reverse transcriptase amplification of cDNA from total RNA was performed using the High-Capacity RNA-to-cDNA Kit (Applied Biosystems), conducted in a PTC-100 thermal cycler (MJ, Research). Taqman Gene Expression Assays (Applied Biosystems) were used in the Applied Biosystems StepOne Plus RT-PCR system to quantify the following target genes during amplification: prodynorphin (Pdyn) Mm00457573_m1, Oprk1 (mouse) Mm01230885_m1, Oprm1 Mm01188089_m1 and the endogenous control Rn18S (mouse) Mm04277571_s1. For each sample the average of the triplicate cycle numbers at threshold crossing $\left(C_{T}\right)$ value for the endogenous control was subtracted from the average $\mathrm{C}_{\mathrm{T}}$ values for the target gene, generating $\Delta \mathrm{C}_{\mathrm{T}}$ values. As the PCR efficiencies for both the endogenous control gene and target gene were equal $(\sim 1)$, the changes in expression of the target gene were expressed as $2-\Delta \Delta \mathrm{C}_{\mathrm{T}}$ for each sample calculated. All data were normalized to the nonstress/saline control group and is expressed as fold change for statistical analysis and presentation.

\section{Statistical Analyses}

Two-way ANOVA were used to determine significant differences between treatments and conditions. Subsequent post hoc tests were conducted with Bonferroni multiple comparisons for behavioral data and with Newman-Keuls multiple comparisons for mRNA expression analysis where appropriate. Student's $t$-test was applied where appropriate. For all tests, $p<0.05$ was considered statistically significant. Data are expressed as mean \pm SEM.

\section{RESULTS}

\section{Consequences of KOR Disruption on BPN's Behavioral Effects in the FST}

The consequences of pretreatment with the KOR antagonist nor-BNI $(10 \mathrm{mg} / \mathrm{kg})$ on BPN's effects in the FST were assessed in WT mice $24 \mathrm{~h}$ after injection (Figure 1a). A significant interaction was observed for nor-BNI pretreatment $\times$ BPN treatment on immobility levels $\left(F_{1,70}=6.440\right.$, $p=0.013)$. Bonferroni multiple comparisons indicated that $\mathrm{BPN}$ was effective in reducing immobility levels in mice
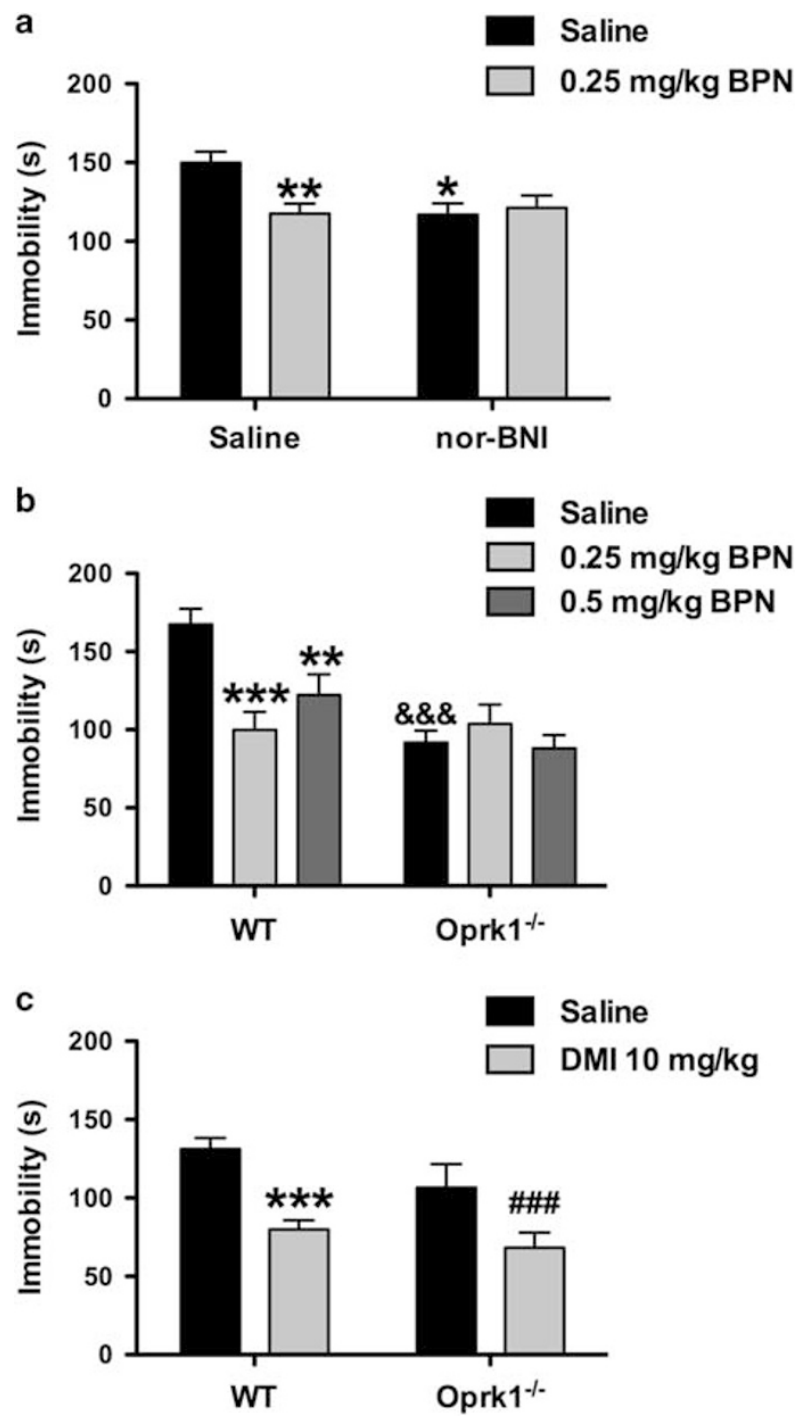

Figure I KOR disruption blocks BPN's behavioral effects in the FST. (a) BPN $(0.25 \mathrm{mg} / \mathrm{kg})$ decreased immobility levels of C57BL/6J mice in the FST but did not further reduce immobility levels in mice pretreated with the KOR antagonist nor-BNI $(10 \mathrm{mg} / \mathrm{kg})$. Saline/Saline, $n=20$; nor-BNI/Saline, $n=15$; Saline/BPN, $n=19$; and nor-BNI/BPN, $n=20$. The asterisks denote where $\mathrm{BPN}$ and nor-BNI, respectively, decreased immobility compared with saline-treated controls ( $* x<0.05$; ** $p<0.0$ I). (b) The ability of BPN $(0.25$ and $0.5 \mathrm{mg} / \mathrm{kg}$ ) to reduce immobility levels of C57BL/6J mice in the FST was blocked in mice with genetic deletion of Oprkl. WT/Saline, $n=20$; WT/ $0.25 \mathrm{mg} / \mathrm{kg}$ BPN, $n=10, W T / 0.5 \mathrm{mg} / \mathrm{kg}$ BPN, $n=10 ; 0 p r k l^{-1-} /$ Saline, $n=17 ;$ Oprk $1^{-1-} / 0.25 \mathrm{mg} / \mathrm{kg}$ BPN, $n=7$; and Oprk $1^{-1-} / 0.5 \mathrm{mg} / \mathrm{kg}$ BPN, $n=10$. The asterisks denote significant differences compared with salinetreated controls $(* * p<0.01$; $* * * *<0.001)$. The symbol \& denotes significant genotype differences between Oprk $I^{-/-}$and WT saline-treated mice $\left({ }^{2} \& \alpha_{p}<0.00 \mathrm{I}\right)$. (c) The tricyclic antidepressant desipramine (DMI, $10 \mathrm{mg} / \mathrm{kg}$ ) reduced immobility scores of WT and Oprk $I^{-1}$ mice compared with saline controls. WT/Saline, $n=19$; WT/DMI, $n=19$; Oprk $\left.\right|^{-1-} /$ Saline, $n=10$; and Oprk $\left.\right|^{-1-} / D M I, n=10$. The asterisks denote a significant difference between DMI and saline-treated WT mice (**** $p<0.00 \mathrm{I})$ and \#\#\# represents a significant difference between DMI and saline-treated Oprk $I^{-1-}$ mice $(p<0.00 I)$. 
pretreated with saline $(p<0.01)$ but not with nor-BNI. NorBNI given alone reduced immobility levels compared with saline/saline-treated controls $(p<0.05)$.

The behavioral effects of BPN in the FST were then assessed in Oprk1 $1^{-/-}$and WT mice (Figure 1b). A significant genotype $\times$ treatment interaction was observed for immobility in the FST $\left(F_{2,69}=6.854, p=0.019\right)$. Pairwise post hoc tests revealed that BPN produced significant reductions of immobility in WT mice at $0.25 \mathrm{mg} / \mathrm{kg}(p<0.001)$ and $0.5 \mathrm{mg} / \mathrm{kg}$ BPN $(p<0.01)$, but these doses were ineffective in Oprk1 $1^{-/-}$mice. In addition, baseline immobility levels were significantly lower in Oprk1 ${ }^{-/-}$mice $(p<0.001)$ compared with WT mice after treatment with saline.

To confirm that KOR-deficient mice could still respond to an antidepressant drug, another cohort of Oprk1 $1^{-/-}$mice and WT mice were treated with the antidepressant desipramine (DMI, $10 \mathrm{mg} / \mathrm{kg}$ ) or saline and tested in the FST 30 min after administration (Figure 1c). A significant main effect of DMI treatment was observed on immobility $\left(F_{1,54}=24.74\right.$, $p=0.001$ ), where DMI reduced immobility in both WT and $O p r k 1^{-/-}$mice. A significant main effect of genotype $\left(F_{1,54}=4.055, p=0.049\right)$, was also observed, where Oprk1 ${ }^{-/-}$ mice exhibited lower immobility scores than WT mice, and no genotype $\times$ treatment interaction.

\section{Consequences of MOR, DOR, and ORL-1 Disruption on BPN's Behavioral Effects in the FST}

To evaluate the role of Oprm1 in BPN's behavioral effects, Oprm $1^{-/-}$mice and their WT littermates were treated with BPN and tested $24 \mathrm{~h}$ later in the FST (Figure 2a). A significant genotype $\times$ treatment interaction was observed for immobility in the FST $\left(F_{2,60}=3.072, p=0.05\right)$. Subsequent post hoc tests revealed that the lowest dose of BPN $(0.125 \mathrm{mg} /$ $\mathrm{kg}$ ) significantly reduced immobility levels only in Oprm $1^{-9-}$ $(p<0.05)$. A higher dose of BPN $(0.25 \mathrm{mg} / \mathrm{kg})$ significantly reduced immobility in both Oprm1 $1^{-/-}$mice $(p<0.001)$ and WT mice $(p<0.05)$.

To evaluate the role of Oprd1 in BPN's behavioral effects, Oprd1 $1^{-1-}$ mice and WT mice were treated with BPN and tested $24 \mathrm{~h}$ later in the FST (Figure 2b). Two-way ANOVA on immobility indicated a significant main effect of BPN treatment $\left(F_{1,49}=22.17, p=0.001\right)$, without a significant treatment $\times$ genotype interaction.

To evaluate the role of the ORL-1 in BPN's behavioral effects, a separate group of WT mice were pretreated with either saline or the ORL-1 antagonist JTC-801 (1 mg/kg) $30 \mathrm{~min}$ prior to BPN $(0.25 \mathrm{mg} / \mathrm{kg})$ and tested $24 \mathrm{~h}$ later in the FST (Figure 2c). Two-way ANOVA revealed a significant main effect of BPN treatment $\left(F_{1,25}=21.76, p=0.001\right)$ and a main effect of pretreatment with JTC-801 $\left(F_{1,25}=4.864\right.$, $p=0.004)$ on immobility scores, without a significant JTC- $801 \times$ BPN interaction.

\section{BPN Reversed UCMS-induced Behavioral Changes}

Mice were randomly assigned to either stress exposure with the UCMS procedure or to the non-stressed control group. All groups were evenly matched in terms of body weight prior to stress exposure, NS saline $=24.5 \pm 0.4 \mathrm{~g}$, $\mathrm{NS} B P N=25 \pm 0.5 \mathrm{~g}$, UCMS Saline $=24.5 \pm 0.5 \mathrm{~g}$ and UCMS BPN $24.6 \pm 0.4 \mathrm{~g}$. As seen in Figure 3a, the UCMS procedure
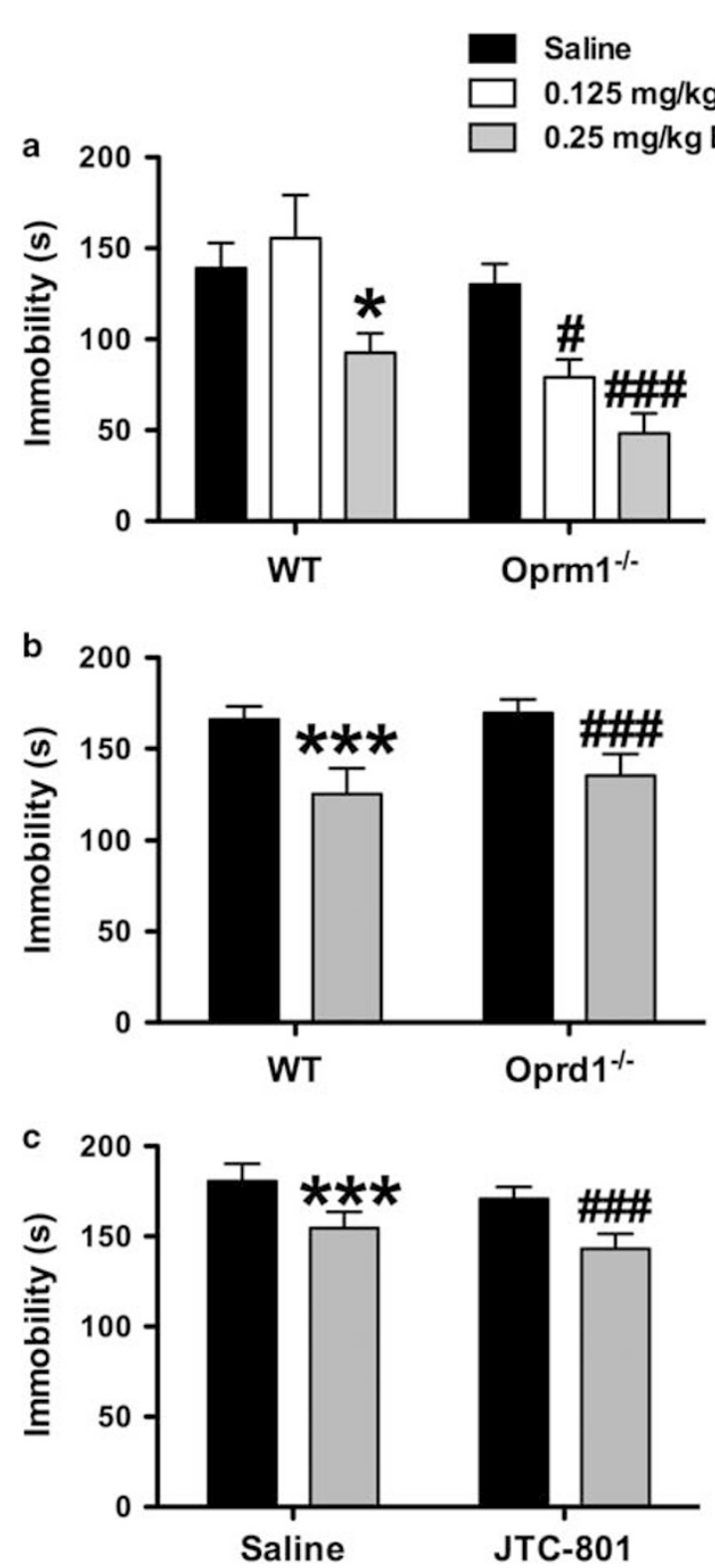

Figure 2 Genetic deletion of Oprm I and OprdI and pharmacological blockade of ORL-I does not inhibit the antidepressant-like effects of BPN in the FST. (a) BPN $(0.25 \mathrm{mg} / \mathrm{kg})$ significantly decreased immobility levels in the FST in WT mice and in mice with genetic deletion of MORs. Furthermore, a subthreshold dose of BPN $(0.125 \mathrm{mg} / \mathrm{kg})$ effectively reduced immobility in Oprm/ ${ }^{-1-}$ mice. WT/Saline, $n=11 ; \mathrm{WT} / 0.125 \mathrm{mg} / \mathrm{kg}$ BPN, $n=8$; WT/ $0.25 \mathrm{mg} / \mathrm{kg}$ BPN, $n=12 ;$ Oprm $l^{-1-} /$ Saline, $n=18 ;$ Oprm $\left.\right|^{-1-} / 0.125 \mathrm{mg} / \mathrm{kg}$ BPN, $n=10$; and Oprm/ $\left.\right|^{-1-} / 0.25 \mathrm{mg} / \mathrm{kg}$ BPN, $n=7$. The asterisks denote significant differences between WT mice treated with BPN $(0.25 \mathrm{mg} / \mathrm{kg})$ and saline $(* p<0.05)$. The symbol \# denotes significant differences between Oprm/ ${ }^{-}$mice treated with BPN and saline $\left({ }^{\#} p<0.05\right.$; \#\#\# $\left.p<0.001\right)$. (b) The ability of BPN $(0.25$ and $0.5 \mathrm{mg} / \mathrm{kg})$ to reduce immobility levels of C57BL/6) mice in the FST was not blocked in mice with a genetic deletion of OprdI, $n=13-14$ per group. The asterisks denote significant differences between WT mice treated with BPN and saline (**** $p<0.00$ I). The symbol \# denotes significant differences between Oprd $I^{-1-}$ mice treated with BPN and saline $\left({ }^{\# \#} p<0.00 \mathrm{I}\right)$. (c) BPN reduced immobility scores in mice pretreated with the ORL-I antagonist JTC-80 I (| mg/kg). N=7-8 mice per group. The symbols denote that BPN treatment significantly decreased immobility in saline-pretreated $(* * * * * 0.00 \mathrm{I})$ and JTC-80I-pretreated

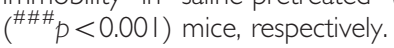


$$
\text { a }
$$

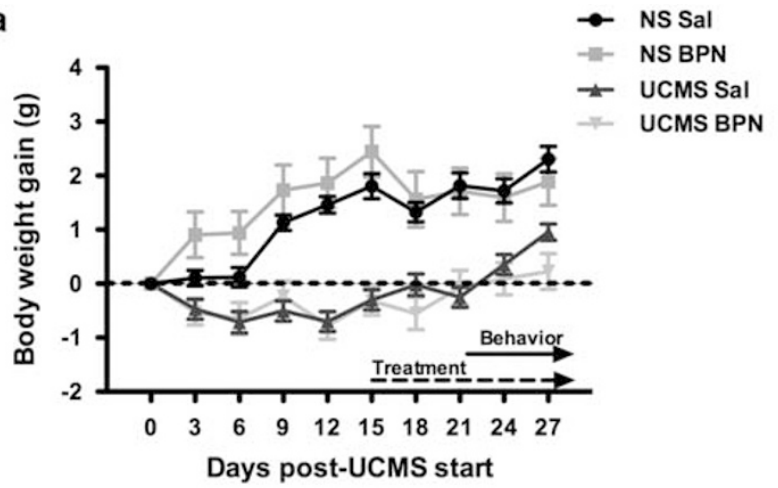

b

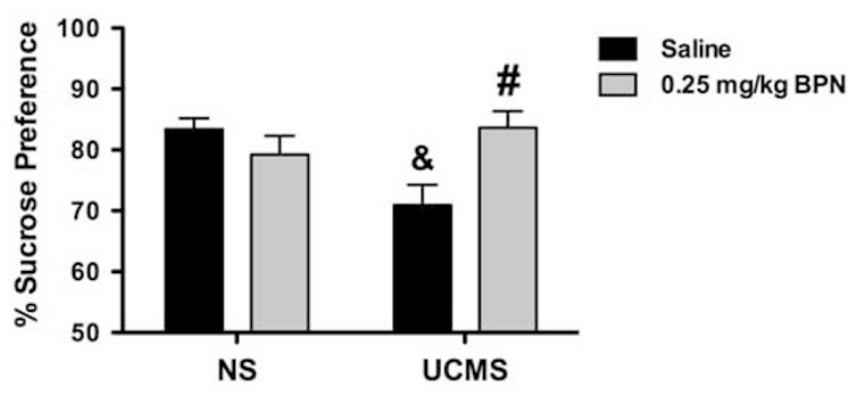

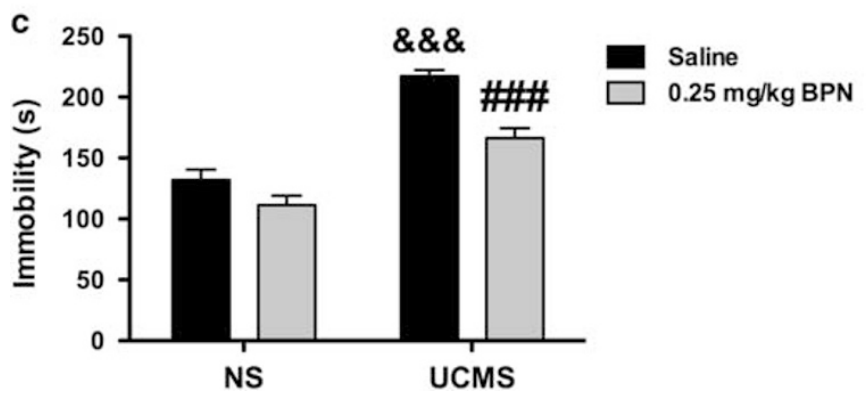

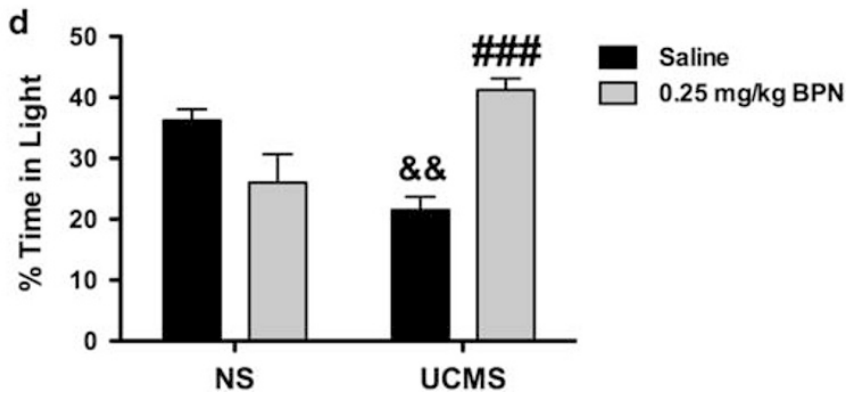

Figure 3 BPN reversed behavioral changes produced by exposure to UCMS. Body weight gain of C57BL/6) mice (a, $n=20$ per group) was suppressed by UCMS compared with NS mice. BPN treatment $(0.25 \mathrm{mg} / \mathrm{kg})$ reversed the UCMS-induced deficits in sucrose preference (b, $n=15-20$ per group), reduced the increased immobility levels of UCMS-exposed mice in the FST (c, $n=19-20$ per group) and increased time spent in light compared with stress mice in the dark/light emergence test ( $d, n=8-10$ per group). The symbol \# denotes significant differences between UCMS-exposed mice given BPN or saline $\left(^{\#} p<0.05\right.$,

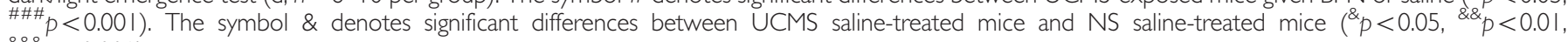
$\& \& \&<0.001)$.

significantly attenuated weight gain in the stressed animals compared with non-stressed controls, as shown by a significant interaction of stress $\times$ day on body weight $\left(F_{9,171}=26.99, p=0.001\right)$. Bonferroni multiple comparison tests revealed that exposure to the stress procedure attenuated weight gain from day 3 through $27(p<0.001)$.

The effect of BPN treatment on sucrose preference in the UCMS procedure is shown in Figure 3b. A significant treatment $\times$ stress interaction was observed on sucrose preference $\left(F_{1,70}=8.342, p=0.005\right)$. Post hoc tests revealed a significant reduction of sucrose preference in UCMSexposed mice but not in non-stressed saline-treated mice $(p<0.05)$. This effect was reversed by BPN treatment $(p<0.05)$.

The effects of BPN treatment on FST performance during the UCMS procedure are shown in Figure 3c. Two-way ANOVA revealed a significant treatment $\times$ stress interaction $\left(F_{1,75}=4.069, p=0.05\right)$ on immobility. Exposure to stress produced a significant increase in immobility compared with non-stressed mice $(p<0.001)$. BPN treatment reversed the stress-induced increase in immobility when compared saline-treated UCMS-exposed mice $(p<0.001)$.

The effects of BPN treatment in the DL emergence test are shown in Figure $3 \mathrm{~d}(n=8-10$ per group). A two-way ANOVA revealed a significant treatment $\times$ stress interaction $\left(F_{1,34}=25.24, p=0.001\right)$ for the percentage of time spent in the light compartment. Subsequent pairwise comparisons revealed that stressed animals spent significantly less time in the illuminated compartment compared with non-stressed controls $(p<0.01)$. BPN treatment significantly reversed this stressed-induced anxiety-like behavior $(p<0.001)$.

\section{Gene Expression of Oprk1, Pdyn, and Oprm1 in Mesocorticolimbic Structures}

Oprk1. In the FC (Figure 4a), a significant treatment $\times$ stress interaction for Oprk1 mRNA expression was observed $\left(F_{1,52}=5.436 p=0.03\right)$. Post hoc tests revealed that Oprk1 mRNA expression was significantly higher in UCMS mice treated with BPN than with saline $(p<0.05)$. A significant treatment $\times$ stress interaction was also observed in the Str $\left(F_{1,71}=6.354, p=0.02\right)$, where UCMS significantly increased expression of Oprk1 ( $p<0.05$, Figure $4 \mathrm{~b})$ and this effect was normalized by BPN treatment $(p<0.05)$. Exposure to stress produced an overall reduction of Oprk1 mRNA expression in the Amy $\left(F_{1,58}=4.769, p=0.04\right.$, Figure $\left.4 c\right)$. No significant changes were measured in the Hp.

Pdyn. A significant treatment $\times$ stress interaction for $P d y n$ mRNA expression was observed in the FC $\left(F_{1,52}=3.723\right.$, $p=0.05$; Figure $4 \mathrm{e})$ and in the $\operatorname{Str}\left(F_{1,71}=4.945 p=0.03\right.$; Figure 4f). Although BPN administration did not alter Pdyn expression in NS mice, drug treatment significantly reversed the stress-induced increase in Pdyn mRNA expression in the FC $(p<0.001)$. Similarly in the Str post-tests revealed a significant decrease in $P d y n$ mRNA expression following $\mathrm{BPN}$ treatment in stress-exposed mice only $(p<0.001)$. No significant alteration in Pdyn mRNA expression was 
FC
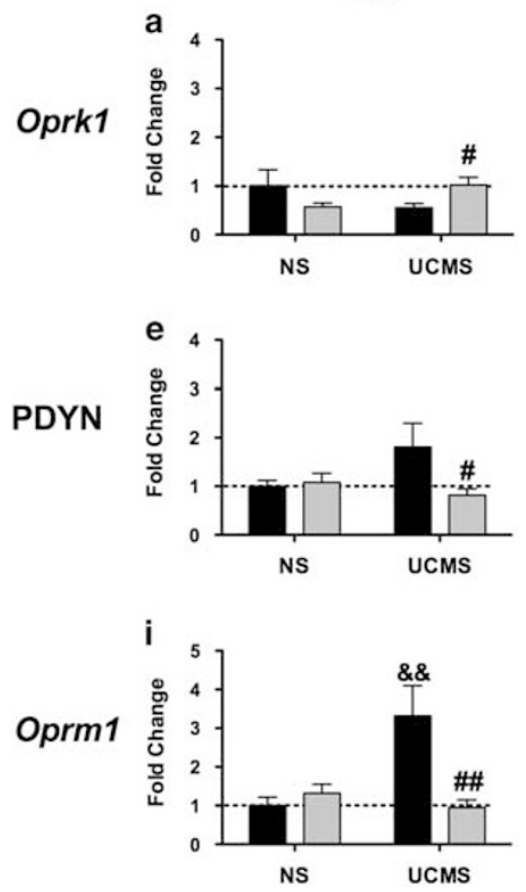

Str
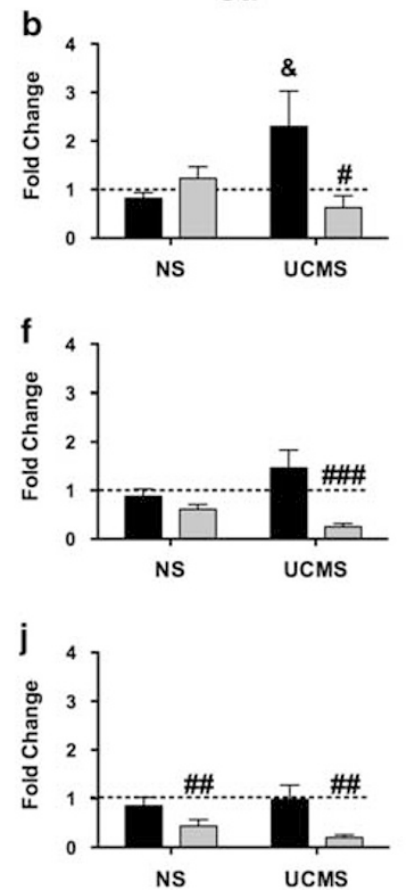

Amy
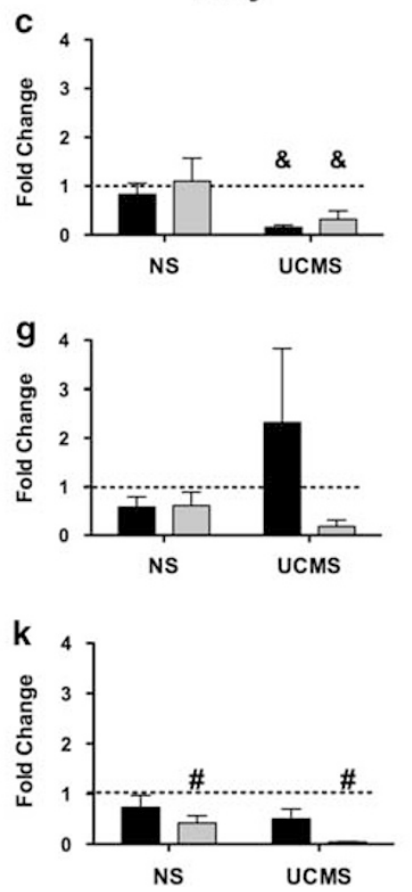

$\mathrm{Hp}$
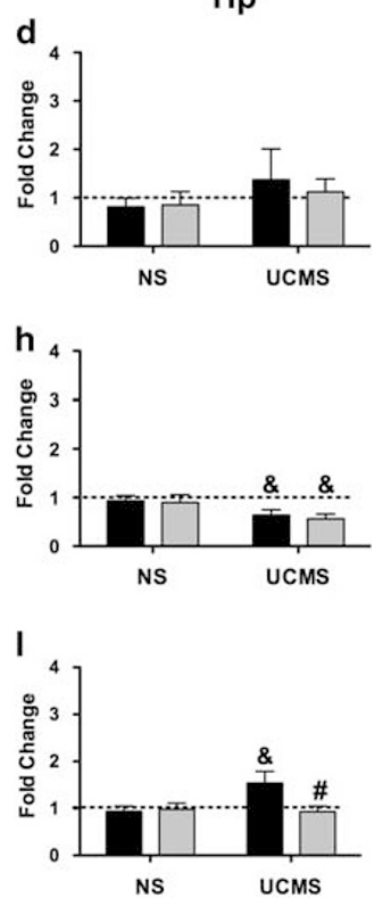

Figure 4 BPN reversed alterations in mesocorticolimbic opioid receptor and prodynorphin gene expression. Alterations in Oprk I mRNA expression in the FC (a, $N=13-14$ per group), Str (b, $N=18-20$ per group), Amy (c, $N=11-19$ per group), and $\mathrm{Hp}$ (d, $N=17-19$ per group); Pdyn mRNA expression in the FC (e), Str (f), Amy (g), and Hp (h); Oprm I mRNA expression in the FC (i), Str (j). Amy (k), and Hp (I). The symbol \# denotes significant differences between BPN-treated mice and their respective saline-treated controls $\# p<0.05, \# p<0.01$, \#\#\# $p<0.001)$. The symbol \& denotes significant differences between UCMS-exposed mice and their respective NS control group ${ }^{\&} p<0.05$, \& ${ }_{p} p<0.01$ ).

observed in the Amy (Figure 4g). However, a significant main effect of stress on Pdyn mRNA expression was evident in the $\operatorname{Hp}\left(F_{1,73}=6.690, p=0.02\right.$; Figure $\left.4 \mathrm{~h}\right)$.

Oprm1. Significant stress $\times$ treatment interactions for Oprm 1 mRNA expression were observed in the FC $\left(F_{1,50}=9.086, \quad p=0.004 ;\right.$ Figure $\left.4 \mathrm{i}\right)$ and in the $\mathrm{Hp}$ $\left(F_{1,72}=3.990, p=0.04\right.$; Figure 41$)$. Oprm 1 mRNA expression was increased by UCMS exposure in both the FC $(p<0.01)$ and Hp $(p<0.05)$ and reversed by BPN treatment in the FC $(p<0.01)$ and Hp $(p<0.05)$. In addition, a significant main effect of BPN treatment was measured for Oprm 1 in the Amy $\left(F_{1,61}=4.366, p=0.04 ;\right.$ Figure 41$)$ and $\operatorname{Str}\left(F_{1,71}=9.664\right.$, $p=0.003)$.

\section{DISCUSSION}

The results of this study present two sets of findings concerning the behavioral effects of BPN that are relevant to affective disorders by using rodent behavioral tests that are sensitive to antidepressant drugs. First, of the potential opioid receptors, the antidepressant-like effects of BPN in the FST are mediated via KORs. Second, utilizing UCMS as a validated procedure for inducing deficits in behavioral domains relevant to depression, we established that chronic BPN reversed deficits in tests for anhedonia, anxiety, and coping with inescapable stress. Furthermore, this is the first report that provides evidence of region-specific alterations in Oprk1, Pdyn, and Oprm1 mRNA expression following UCMS that are reversed by BPN and may underlie the behavioral effects of this KOR antagonist. Taken together, these data provide preclinical evidence that supports recent clinical studies of BPN as a novel therapeutic for depression.

Previously, our laboratory (Browne et al, 2015; Falcon et al, 2015) and others (Almatroudi et al, 2015) have shown that BPN reduces immobility in the FST in different strains of mice and rats, a test sensitive to the effects of different classes of antidepressant drugs. As BPN has activity at multiple opioid receptors (Lutfy and Cowan et al, 2004), an important goal of this study was to identify which opioid receptors were associated with BPN's effects in the FST. Here, we determined that BPN's effects in the FST are mediated via KORs. Pharmacological blockade of KORs with the long-lasting antagonist nor-BNI and genetic deletion of KORs $\left(\right.$ Oprk1 $\left.{ }^{-9-}\right)$ prevented the effects of BPN. In our study, reduced KOR signaling diminished immobility levels in the FST compared with controls. A previously published study reported no change in the FST performance of Oprk1 $1^{-/}$ mice (Filliol et al, 2000), although these differences may be attributed to procedures or genetic background. Despite a phenotype of reduced baseline immobility, Oprk1 $1^{-1-}$ mice were still sensitive to the behavioral effects of the noradrenergic antidepressant desipramine in the FST, confirming that blockade of BPN's effects in Oprk1 $1^{-/-}$mice was not due to a floor effect. Thus, BPN probably could not produce 
additive effects on FST immobility levels if KORs were already occupied by nor-BNI or compromised genetically. In contrast, genetic deletion of MORs $\left(\mathrm{Oprm}^{-/-}\right)$, resulted in shifting the dose-response curve to the left, where Oprm1 $1^{-/-}$mice showed increased sensitivity to the antidepressant-like effects of BPN. These data, in addition to the previously reported inability of the MOR antagonist clocinnamox to block the effects of BPN (Almatroudi et al, 2015), further support the hypothesis that BPN elicits its behavioral effects in the FST via KOR rather than MOR antagonism. Furthermore, genetic deletion of DORs $\left(O p r d 1^{-/-}\right)$and blockade of ORL-1 receptors with JTC-801 did not block or augment the effects of BPN in the FST. Oprd1 $1^{-1-}$ mice have been reported to display a higher baseline immobility compared with controls (Filliol et al, 2000), although we did not replicate this elevation in baseline immobility levels in this study. Nevertheless, the ability of BPN to produce antidepressant-like effects in these mice was still evident. BPN has low affinity for ORL-1 receptors, and we did not observe diminished activity of BPN's effects in the FST following systemic administration of JTC-801. At this dose JTC-801 is known to diminish allodynia and hyperalgesia in mice and reverse stress-induced behavioral deficits (Delaney et al, 2012; Yamada et al, 2002; Zhang et al, 2015). Numerous studies have shown that activation of ORL-1 receptors produces anxiolytic effects, whereas antagonism evokes antidepressant-like responses (Witkin et al, 2014). As such, future studies may evaluate BPN's effects for involvement of ORL-1 receptors following pretreatment with both agonists and antagonists using a broader range of behavioral tests. Overall these data suggest that BPN's effects at DOR and ORL-1 receptors do not modulate its behavioral effect in the FST. One caveat of these studies was variability in baseline immobility values across groups of control WT mice. One likely reason for this variability between groups is timing of the injections. In the case of the DMI study, the injections occurred $30 \mathrm{~min}$ prior to testing; it was these animals that exhibited the lowest levels of baseline immobility (130 s). Nevertheless, BPN consistently resulted in a decrease of baseline immobility of $>25 \%$ across all studies, except when tested in KOR knockouts and norBNI-treated mice where the effect was blocked. Taken together these studies imply that KOR antagonism is necessary and sufficient to induce antidepressant-like effects in the FST.

In the present study, we evaluated BPN's effects in mice after exposure to UCMS, a rodent model that induced deficits in multiple behavioral domains relevant to depression. The core features of UCMS exposure include anhedonia, prodepressive, and anxiety-like behavior, in addition to physiological alterations in body weight, stress hormones, and sleep architecture (Willner, 2005). In this study, UCMS produced significant reductions in body weight gain, marked anhedonia as measured by sucrose preference, increased prodepressive behavior in the FST, and decreased exploration of a brightly lit arena in the dark/light emergence test. Administration of BPN reversed these behavioral deficits caused by UCMS when mice were tested after only 7 days following the onset of treatment. Typically, these behavioral deficits are reversed only following chronic (21-28 days) treatment with conventional antidepressant drugs (Willner, 2005). Compared with this expected time course, BPN may be a more rapidly acting antidepressant compound. These are the first data to highlight BPN's efficacy in a validated rodent model of depression.

Exposure to UCMS and chronic BPN treatment induced region-specific alterations in the mRNA expression of Oprk1, Oprm1, and Pdyn, highlighting a potentially important role of cortico-striatal opioid signaling in the treatment of depression. The exposure to chronic stress produced significant changes in opioid gene expression in a number of regions (Oprk1 increased in Str and decreased in Amy; reduced Pdyn in HP; and increased Oprm1 in FC and Hp). Interestingly, BPN reversed the effects of chronic stress in some regions (Oprk1 in FC and Str; Pdyn in FC and Str; Oprm1 in FC and $\mathrm{Hp}$ ) pointing to potential functional interactions between stress and drug treatment. Opioid gene expression was altered by BPN treatment irrespective of stress in only two regions (Oprm1 in Str and Amy). This suggests, that for most brain regions, exposure to environmental stress and the associated release of endogenous opioid peptides altered the pharmacological effects of BPN.

These findings can be seen as in line with neuroimaging studies in depressed patients, which have highlighted low KOR availability in Amy-anterior cingulate cortex-ventral striatal circuitry in the phenotypic expression of dysphoria (Pietrzak et al, 2014) and aberrant MOR signaling in the cortical-amygdalar circuitry in the response to aversive stimuli (Kennedy et al, 2006; Liberzon et al, 2002). They also agree in part with the results of opioid gene expression in post-mortem studies of suicide victims (Hurd et al, 1997). Although the data presented in this study exposes and confirms significant reductions in opioid gene expression post stress or drug treatment, their correlative nature cannot yet implicate any mechanism in mediating BPN's behavioral effects. Instead, they form a new framework for studies using concerted efforts targeting the specific neurocircuitry mediating the association between opioid signaling and affective behavior to provide functional evidence linked to behavior changes after chronic stress.

Clinical evidence continues to show that BPN has considerable promise for treating affective disturbances in treatment-resistant patients (Bodkin et al, 1995; Ehrich et al, 2015; Karp et al, 2014; Nyhuis et al, 2008). Preclinical experiments allow the effects of $\mathrm{BPN}$ to be characterized in a context relevant to affective behavior using tests involving stress that are also sensitive to the effects of antidepressant drugs. In the present study, BPN was confirmed to reduce immobility in the FST (Falcon et al, 2015; Browne et al, 2015). Further studies evaluating the mechanism of action mediating these effects, using pharmacological antagonists and genetic deficiency, showed that reduced KOR signaling likely accounts for the effects of $\mathrm{BPN}$ in the FST. In addition, the antidepressant-like effects of BPN were shown in the UCMS model involving behavioral deficits measured after more rigorous exposure to stress and repeated treatment with $\mathrm{BPN}$. The results encourage further investigations into the clinical effects of $\mathrm{BPN}$ relevant to affective behavior. Moreover, identification of the mechanisms underlying the effects of BPN may lead to testing novel treatments, such as KOR antagonists, that may be effective antidepressants without the potential liabilities of BPN. 


\section{FUNDING AND DISCLOSURE}

IL was a consultant for Alkermes in 2013. The authors have no other conflicts to disclose.

\section{ACKNOWLEDGMENTS}

We would like to acknowledge Summer Undergraduate Internship Program at the University of Pennsylvania, from which both RML and VCF received support. This research was supported by US public health services (USPHS) grants R01 MH92412, P30 DA013429 and T32 MH14654.

\section{REFERENCES}

Almatroudi A, Husbands SM, Bailey CP, Bailey SJ (2015). Combined administration of buprenorphine and naltrexone produces antidepressant-like effects in mice. J Psychopharmacol 29: 812-821.

Balu DT, Hodes GE, Anderson BT, Lucki I (2009). Enhanced sensitivity of the MRL/MpJ mouse to the neuroplastic and behavioral effects of chronic antidepressant treatments. Neuropsychopharmacology 34: 1764-1773.

Bodkin JA, Zornberg GL, Lukas SE, Cole JO (1995). Buprenorphine treatment of refractory depression. Clin Psychopharmacology 15: 49-57.

Browne CA, van Nest DS, Lucki I (2015). Antidepressant-like effects of buprenorphine in rats are strain dependent. Behav Brain Res 278C: $385-392$.

Carlezon WA Jr., Beguin C, DiNieri JA, Baumann MH, Richards MR, Todtenkopf MS et al (2006). Depressive-like effects of the kappa-opioid receptor agonist salvinorin A on behavior and neurochemistry in rats. J Pharmacol Exp Ther 316: 440-447.

Carr GV, Bangasser DA, Bethea T, Young M, Valentino RJ, Lucki I (2010). Antidepressant-like effects of kappa-opioid receptor antagonists in Wistar Kyoto rats. Neuropsychopharmacology 35: 752-763.

Cowan A (2007). Buprenorphine: the basic pharmacology revisited. J Addict Med 1: 68-72.

Delaney G, Dawe KL, Hogan R, Hunjan T, Roper J, Hazell G et al (2012). Role of nociceptin/orphanin FQ and NOP receptors in the response to acute and repeated restraint stress in rats. J Neuroendocrinol 24: 1527-1541.

Ehrich E, Turncliff R, Du Y, Leigh-Pemberton R, Fernandez E, Jones R et al (2015). Evaluation of opioid modulation in major depressive disorder. Neuropsychopharmacology 40: 1448-1455.

Falcon E, Maier K, Robinson SA, Hill-Smith TE, Lucki I (2015). Effects of buprenorphine on behavioral tests for antidepressant and anxiolytic drugs in mice. Psychopharmacology 232: 907-915.

Filliol D, Ghozland S, Chluba J, Martin M, Matthes HW, Simonin F et al (2000). Mice deficient for delta- and mu-opioid receptors exhibit opposing alterations of emotional responses. Nat Genet 25: 195-200.

Hurd YL, Herman MM, Hyde TM, Bigelow LB, Weinberger DR, Kleinman JE (1997). Prodynorphin mRNA expression is increased in the patch vs matrix compartment of the caudate nucleus in suicide subjects. Mol Psychiatry 2: 495-500.

Ipser JC, Terburg D, Syal S, Phillips N, Solms M, Panksepp J et al (2013). Reduced fear-recognition sensitivity following acute buprenorphine administration in healthy volunteers. Psychoneuroendocrinology 38: 166-170.

Karp JF, Butters MA, Begley AE, Miller MD, Lenze EJ, Blumberger DM et al (2014). Safety, tolerability, and clinical effect of low-dose buprenorphine for treatment-resistant depression in midlife and older adults. J Clin Psychiatry 75: e785-e793.
Kennedy SE, Koeppe RA, Young EA, Zubieta JK (2006). Dysregulation of endogenous opioid emotion regulation circuitry in major depression in women. Arch Gen Psychiatry 63: 1199-1208.

Land BB, Bruchas MR, Lemos JC, Xu M, Melief EJ, Chavkin C (2008). The dysphoric component of stress is encoded by activation of the dynorphin kappa-opioid system. J Neuroscience 28: 407-414.

Liberzon I, Zubieta JK, Fig LM, Phan KL, Koeppe RA, Taylor SF (2002). mu-Opioid receptors and limbic responses to aversive emotional stimuli. Proc Natl Acad Sci USA 99: 7084-7089.

Lucki I, Dalvi A, Mayorga AJ (2001). Sensitivity to the effects of pharmacologically selective antidepressants in different strains of mice. Psychopharmacology 155: 315-322.

Lutfy K, Cowan A (2004). Buprenorphine: a unique drug with complex pharmacology. Curr Neuropharmacol 2: 395-402.

Lutz PE, Kieffer BL (2013). Opioid receptors: distinct roles in mood disorders. Trends Neurosci 36: 195-206.

Mague SD, Pliakas AM, Todtenkopf MS, Tomasiewicz HC, Zhang Y, Stevens WC Jr. et al (2003). Antidepressant-like effects of kappa-opioid receptor antagonists in the forced swim test in rats. J Pharmacol Exp Ther 305: 323-330.

McLaughlin JP, Marton-Popovici M, Chavkin C (2003). Kappa opioid receptor antagonism and prodynorphin gene disruption block stressinduced behavioral responses. J Neuroscience 23: 5674-5683.

Mozhui K, Karlsson RM, Kash TL, Ihne J, Norcross M, Patel S et al (2010). Strain differences in stress responsivity are associated with divergent amygdala gene expression and glutamate-mediated neuronal excitability. J Neuroscience 30: 5357-5367.

Nyhuis PW, Gastpar M, Scherbaum N (2008). Opiate treatment in depression refractory to antidepressants and electroconvulsive therapy. J Clin Psychopharmacology 28: 593-595.

Pietrzak RH, Naganawa M, Huang Y, Corsi-Travali S, Zheng MQ, Stein MB et al (2014). Association of in vivo kappa-opioid receptor availability and the transdiagnostic dimensional expression of trauma-related psychopathology. JAMA Psychiatry 71: 1262-1270.

Porsolt RD, Bertin A, Jalfre M (1977). Behavioral despair in mice: a primary screening test for antidepressants. Arch Int Pharmacodyn Ther 229: 327-336.

Schmidt HD, Duman RS (2010). Peripheral BDNF produces antidepressant-like effects in cellular and behavioral models. Neuropsychopharmacology 35: 2378-2391.

Strekalova T, Steinbusch HW (2010). Measuring behavior in mice with chronic stress depression paradigm. Prog Neuropsychopharmacol Biol Psychiatry 34: 348-361.

Syal S, Ipser J, Terburg D, Solms M, Panksepp J, Malcolm-Smith S et al (2015). Improved memory for reward cues following acute buprenorphine administration in humans. Psychoneuroendocrinology 53: 10-15.

Willner P (1997). Validity, reliability and utility of the chronic mild stress model of depression: a 10-year review and evaluation. Psychopharmacology 134: 319-329.

Willner P (2005). Chronic mild stress (CMS) revisited: consistency and behavioural-neurobiological concordance in the effects of CMS. Neuropsychobiology 52: 90-110.

Witkin JM, Statnick MA, Rorick-Kehn LM, Pintar JE, Ansonoff M, Chen Y et al (2014). The biology of Nociceptin/Orphanin FQ (N/OFQ) related to obesity, stress, anxiety, mood, and drug dependence. Pharmacol Ther 141: 283-299.

Yamada H, Nakamoto H, Suzuki Y, Ito T, Aisaka K (2002). Pharmacological profiles of a novel opioid receptor-like1 (ORL (1)) receptor antagonist, JTC-801. Br J Pharmacol 135: 323-332.

Zhang Y, Simpson-Durand CD, Standifer KM (2015). Nociceptin/ orphanin FQ peptide receptor antagonist JTC-801 reverses pain and anxiety symptoms in a rat model of post-traumatic stress disorder. Br J Pharmacol 172: 571-582.

Supplementary Information accompanies the paper on the Neuropsychopharmacology website (http://www.nature.com/npp) 